\title{
Enhancing Nonlinear Interactions by the Superposition of Plasmonic Lattices on $\chi^{(2)}$-Nonlinear Photonic Crystals
}

\author{
Alejandro Gómez-Tornero, Pablo Palacios, Pablo Molina, Sol Carretero-Palacios, Luisa E. Bausá, \\ and Mariola O Ramírez*
}

Cite This: ACS Photonics 2021, 8, 2529-2537

Read Online

\section{ACCESS I}

山l Metrics \& More

Article Recommendations

Supporting Information

ABSTRACT: Plasmonic structures have been revealed as efficient units to enhance localized nonlinear phenomena generated at dielectric-metal interfaces. However, their effect on the nonlinear interactions provided by quasi-phase matching processes in $\chi^{(2)}$ modulated dielectric crystals have been scarcely addressed, mainly due to the complexity in manufacturing appropriate periodic plasmonic structures overlying the $\chi^{(2)}$ dielectric structure. Here, by a simple method we have fabricated a periodic structure based on the combination of two commensurate lattices: a periodic lattice of chains of $\mathrm{Ag}$ nanoparticles and a periodic lattice of $\chi^{(2)}$-modulation based on a ferroelectric domains structure. The hybrid system supports multiple surface plasmon lattice resonances (SLRs) at the technologically relevant NIR spectral region, which yield the enhancement of the nonlinear diffraction pattern generated by the $\chi^{(2)}$ structure. The superposition of the plasmonic and the $\chi^{(2)}$-modulation lattice results in a 20 -fold enhancement of the directional SHG due to the excitation of SLRs by the interacting waves involved in the nonlinear process. The results are obtained in lithium niobate, a widely used crystal in optoelectronics, and demonstrate the potential of the approach to design integrated solidstate platforms for on-chip optical steering, multiplexing or quantum technologies.

KEYWORDS: quasi-phase matching, nonlinear optics, surface lattice resonances, domain engineering, LiNbO 3 , plasmonic lattices

ince the first demonstration of nonlinear (NL) diffraction $\checkmark$ by I. Freud in $1968{ }^{1}$ ferroelectric domain structures have been used to manipulate and control nonlinear optical interactions in monolithic devices. These structures, generally known as nonlinear photonic crystals (NLPC), exhibit a periodic spatial modulation of the sign of the second order nonlinear susceptibly, $\chi^{(2)}$, allowing different quasi-phase matching (QPM) NL processes. ${ }^{2-4}$ The periodic modulation of $\chi^{(2)}$ can be achieved in $1 \mathrm{D}, 2 \mathrm{D}$, and 3D geometries, resulting in a variety of phase matching schemes to simultaneously control the spatial and spectral distribution of multiphoton processes. $^{5-9}$ NL generation and manipulation of Airy beams, ${ }^{10}$ mirrorless optical parametric oscillation, ${ }^{11}$ simultaneous generation of second to fifth harmonic beams with conical spatial distribution, ${ }^{12}$ or on-chip spatial control of entangled photons ${ }^{13}$ are some examples of the capability of NLPCs. However, novel strategies are needed to improve the efficiency of the NL response in $\chi^{(2)}$-based miniaturized devices.

In this context, the association of metallic nanostructures with nonlinear dielectric crystals has provided an interesting approach to improve the efficiency of frequency conversion processes at the nanoscale. ${ }^{14-17}$ In particular, the extraordinary capability of localized surface plasmon resonances (LSP) for field confinement has been recently exploited to significantly enhance quadratic nonlinear phenomena localized at the metal-nonlinear dielectric interfaces. ${ }^{18-20}$ However, to our knowledge, the collective effect of periodic arrays of metal structures on the NL processes generated by dielectric NLPCs has been little explored. In addition to the localized enhancement of the NL response at the metal-nonlinear dielectric interface, plasmonic lattices placed on the surface of ferroelectric domain patterns can allow the interaction of plasmonic surface lattice resonances (SLRs) with the waves participating in the NL processes generated at the $\chi^{(2)}$ dielectric crystal. This can lead to new integrated NL platforms for enhancing and shaping light in specific directions, as required for scientific and technological applications.

In the last years, the use of arrays of plasmonic nanoparticles (NPs) supporting collective SLRs has proven to be an interesting approach for manipulating light-matter interac-

Received: May 26, 2021

Published: July 22, 2021 
a
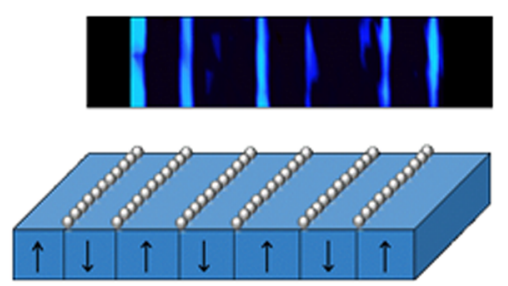

C

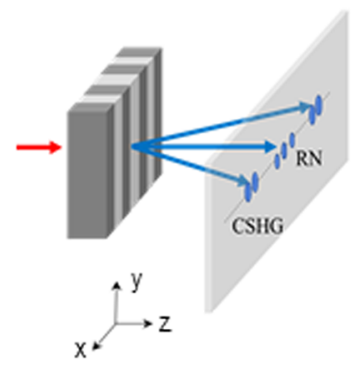

e b
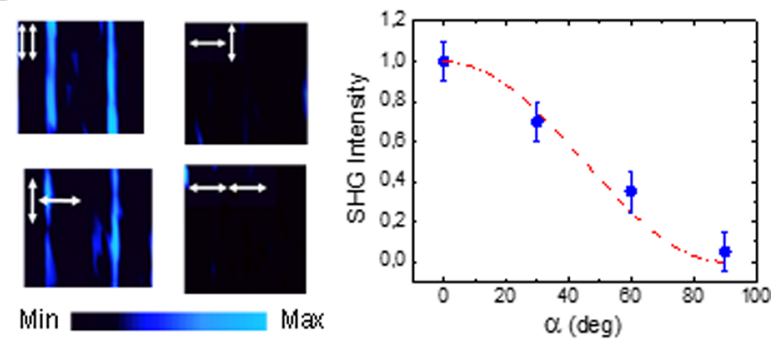

d
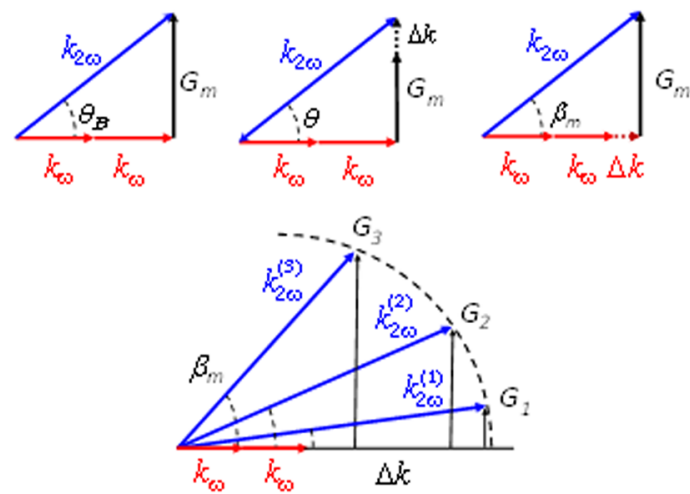

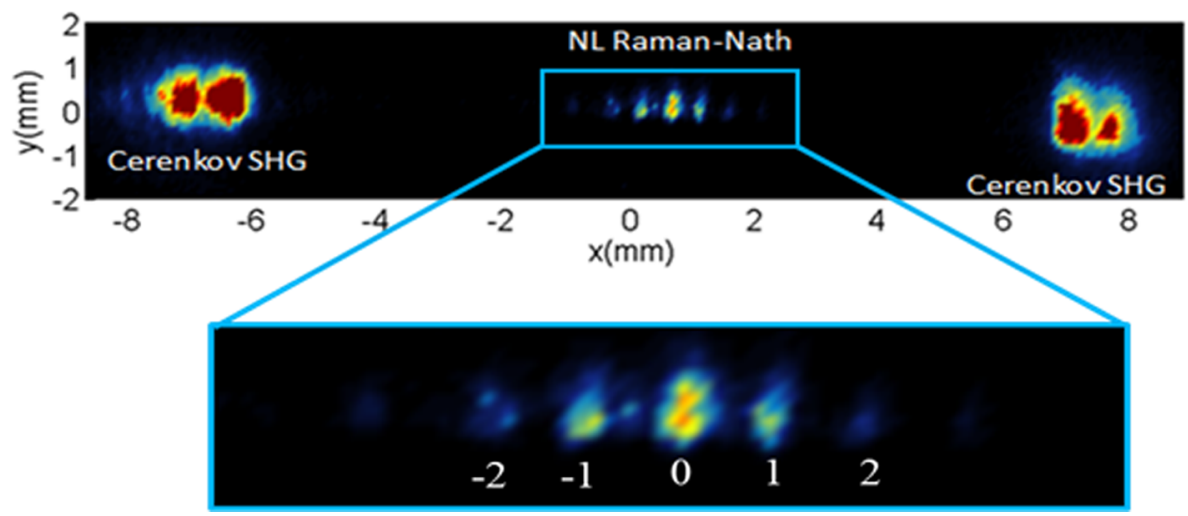

Figure 1. 1D hybrid plasmonic-NLPC. (a) Schematics of the fabricated structure showing the periodical arrangement of linear chains of Ag NPs on the domain boundary surfaces of a PPLN. Top panel: spatially resolved confocal SHG image. (b) Polarization dependence of the localized SHG enhancement produced by the plasmonic chains. Left and center panels: spatial distribution of the SHG intensity of a region containing two chains of Ag NPs. The arrows indicate the orientation of the polarization of the fundamental beam and emitted SHG, respectively. Right panel: normalized SHG integrated intensity as a function of the polarization angle, $\alpha$, of the fundamental beam $\left(\alpha=0^{\circ}\right.$ corresponds to light polarized parallel to the plasmonic chains). Dashed red line represents the $\cos ^{2} \theta$ dependence. (c) Schematics of the noncollinear SHG process for a fundamental beam propagating along the ferroelectric axis. The NL RN diffraction and CSHG are indicated. (d) From left to right, phase matching diagrams for nonlinear Bragg diffraction, CSHG, and NL RN diffraction. The bottom panel represents the NL RN diffraction for different orders. (e) Projection of the NL patterns displaying the simultaneous generation of CSHG (external spots) and the NL RN diffraction (central spots). The expanded view shows a detail of the NL RN diffraction pattern. The numbers indicate the NL diffraction orders $(m)$. The experiments were carried out at normal incidence for a fundamental beam of $800 \mathrm{~nm}$.

tions with applications in a diversity of fields such as lasing, biosensing, solid-state lighting, or photovoltaics. ${ }^{21-27}$ They have also been employed for second harmonic generation (SHG) experiments by using different design strategies. ${ }^{28-32}$ However, most of the plasmonic arrangements use single NPs, and little attention has been paid to the study of complex structures arranged in periodical patterns. This fact, along with the lack of studies on hybrid systems analyzing the association of plasmonic lattices with $\chi^{(2)}$-based NL materials, limits the current impact of NL response in nanophotonic structures.

Here, we propose a hybrid plasmonic-dielectric architecture that combines plasmonic superlattices with dielectric NLPCs to generate, enhance, and control the macroscopic nonlinear response of the NLPCs. In particular, we investigate the effect of SLRs supported by periodic arrays of linear chains and hexagonal necklaces of Ag NPs on the NL diffraction pattern of NLPCs based on periodically poled lithium niobate, a widespread used dielectric material in advanced photonics and optoelectronics. ${ }^{33-36}$ On the one hand, we experimentally and theoretically demonstrate the presence of multiple SLRs in the near-infrared (NIR) spectral region due to the collective optical response sustained by periodical arrays of linear chains of interacting Ag NPs.

On the other hand, by the superposition of the plasmonic superlattice on the domain engineered $\chi^{(2)}$ modulated ferroelectric crystal (NLPC), we demonstrate a noticeable enhancement and beaming of the SHG due to the excitation of collective SLRs by the interacting waves involved in the NL 
process. The work provides a viable approach to manipulate quasi-phase matched NL diffraction processes in miniaturized photonic devices, enabling new avenues for shaping multiwavelength up-conversion processes.

In this work, we first analyze the effect of the LSP resonance of a single plasmonic unit (linear chain of interacting Ag NPs) on the SHG by the $\mathrm{LiNbO}_{3}$ crystal at the metal-dielectric interface. Due to the coupling of the fundamental radiation with the LSP mode of a single metallic chain, a localized SHG enhancement of around 2 orders of magnitude is observed. Then, the spectral and angular dependence of the NL diffraction pattern generated by the NLPC is analyzed in the presence of the periodical array of plasmonic chains. In this case, the SLRs supported by the plasmonic lattice produce the enhancement of the fundamental radiation, resulting in a remarkable 20-fold enhancement of the macroscopic SHG at the directions imposed by the NLPC.

Finally, to emphasize the potential of hybrid plasmonicdielectric superlattices for tailoring NL diffraction processes, we extend the study to a $2 \mathrm{D}$ geometry by using a square lattice of hexagonal plasmonic necklaces of Ag NPs deposited on the domain boundary surfaces of a $2 \mathrm{D}$ ferroelectric domain pattern. The NL QPM generalization from $1 \mathrm{D}$ to $2 \mathrm{D}-\chi^{(2)}$ modulation expands the access to additional reciprocal lattice vectors steering the enhanced radiated waves into different directions. The results of this work demonstrate the capability of SLRs to modify the nonlinear interactions in a $\chi^{(2)}$-nonlinear dielectric material, enabling alternative paths for light shaping into well-defined SHG beams.

\section{RESULTS AND DISCUSSION}

Periodically poled $\mathrm{LiNbO}_{3}$ crystals (PPLN) are extensively used 1D NLPCs for efficient frequency conversion processes, including multiharmonic generation, optical parametric oscillation, and nonlinear diffraction. ${ }^{37-39}$ Additionally, ferroelectric domain patterns in these crystals can be used as templates for domain-selective photodeposition of metallic NPs on its surface. This enables effective-cost fabrication of hybrid plasmonic-nonlinear dielectrics by means of ferroelectric lithography. ${ }^{40}$ Figure 1a shows a scheme of the 1D hybrid material used for this study. A PPLN crystal with a $\chi^{(2)}$ spatial modulation of poling period $\Lambda=6.2 \mu \mathrm{m}$ is used as a NLPC. On top of it, a plasmonic lattice of linear chains of $\mathrm{Ag}$ NPs is fabricated at the domain boundaries surfaces by the aforementioned ferroelectric lithography technique. The spatial period of the plasmonic lattice is $\Lambda^{\prime}=3.1 \mu \mathrm{m}$. Note that the $\chi^{(2)}$ period is $\Lambda=2 \Lambda^{\prime}$. Therefore, we have fabricated a hybrid plasmonic-nonlinear dielectric periodic structure based on the combination of two commensurate lattices.

The plasmonic chains are composed of quasi-spherical Ag NPs with an average size of around $55 \mathrm{~nm}$, spaced around 2 $\mathrm{nm}$. See Materials and Methods for experimental fabrication details. SEM of the plasmonic structures and dark field images can be found in Figure S1 of the Supporting Information. The optical response of a single metallic chain has been reported elsewhere. ${ }^{41-43}$ It is characterized by two main modes, a transverse (polarization perpendicular to the plasmonic chain) and spectrally narrow mode centered at around $400 \mathrm{~nm}$, and a longitudinal one (polarization parallel to the chain) centered at around $600 \mathrm{~nm}$, which extends from the visible to the NIR. Their corresponding absorption and scattering cross-section spectra are shown in Figure S2a and b, respectively. On the other hand, as illustrated in Figure S2c, the amplitude of the near-field is remarkably larger for the longitudinal chain plasmon mode, showing intense hot spots localized between the NPs. ${ }^{44}$

Figure 1a shows a spatial map of the SHG intensity on the surface of the sample obtained by confocal microscopy. As observed, a significant localized enhancement of the SHG is obtained when the fundamental beam is focused at the immediacy of the plasmonic chains. Indeed, to achieve a comparable SHG intensity from the bare $\mathrm{LiNbO}_{3}$ substrate, the incident power of the fundamental beam has to be increased by 1 order of magnitude. Thereby, considering the quadratic dependence of the SHG intensity on the fundamental beam, a 100 -fold SHG enhancement is estimated to occur at the metal-NL dielectric interface, which points out the key role of hybrid plasmonic-nonlinear dielectrics to boost the efficiency of NL processes at subwavelength scales. ${ }^{14}$ The polarization dependence of the enhanced SHG in the proximities of the metallic chains has been analyzed for different wavelengths and polarization configurations of the fundamental and SHG beams. As shown in Figure 1b, the relevant feature is the polarization of the fundamental radiation, regardless of the polarization state of the nonlinear generated waves. The SHG enhancement displays the highest value when the incident light is polarized parallel to the chains $\left(\alpha=0^{\circ}\right)$, decreasing when the polarization angle is increased. Minimum values of the SHG occur when the fundamental beam is polarized perpendicular to the chain axis $\left(\alpha=90^{\circ}\right)$. These results are consistent with the much larger field enhancement displayed by the longitudinal chain plasmon mode with respect to that of the transversal mode and point out the local field enhancement of the fundamental radiation as the dominant mechanism of the plasmon-enhanced SHG.

Before studying the effect of SLRs provided by the arrays of linear chains on the SHG, we analyze the NL diffraction patterns generated at normal incidence by our NLPC in the absence of plasmonic chains. The sample was oriented with the $\chi^{(2)}$-modulation lattice vector perpendicular to the fundamental beam. The generated NL response was projected onto a screen as sketched in Figure 1c. In this configuration, three different NL diffraction phenomena can be distinguished depending on the type of QPM condition (Figure 1d). The first one is the so-called nonlinear Bragg diffraction, which occurs when the full vectorial phase matching condition $2 k_{\omega}+$ $m \mathbf{G}_{0}=k_{2 \omega}$ is satisfied, $k_{\omega}$ and $k_{2 \omega}$ being the wave vectors of the fundamental and second harmonic waves, respectively, $m$ is an integer number related to the diffraction order, and $\mathbf{G}_{\mathbf{0}}$ is the reciprocal lattice vector of the $\chi^{(2)}$ modulation $\left(\mathbf{G}_{0}=2 \pi / \Lambda\right)$. From here, two partial momentum conservation conditions, which can be independently satisfied, can be derived. These correspond to the longitudinal and transverse momentum conservation, which provide the NL processes known as Cerenkov-type SHG (CSHG) and NL Raman-Nath (RN) diffraction, respectively. Figure 1e displays the simultaneous generation of these two processes in our NLPC. The two peripheral spots appearing at $66^{\circ}$ and $68^{\circ}$ correspond to CSHG. Their angular position is determined by the refractive index of the uniaxial $\mathrm{LiNbO}_{3}$ crystal and does not depend on the lattice period. ${ }^{45}$ The diffracted spots observed at the central region of the pattern correspond to a multiorder NL RN diffraction. In this case, the deflected angles are determined by the transverse QPM condition, $k_{2 \omega} \sin \beta_{m}=m G_{0}$, where $\beta_{m}$ defines the angular position of the $m$-th NL diffraction order. $^{46,47}$ The external angles of the RN diffracted spots were 
a
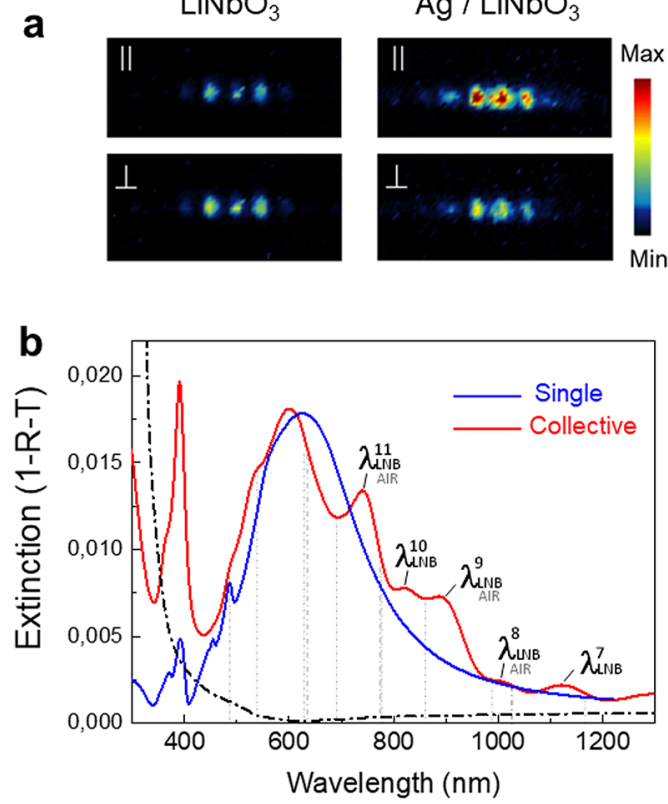

C
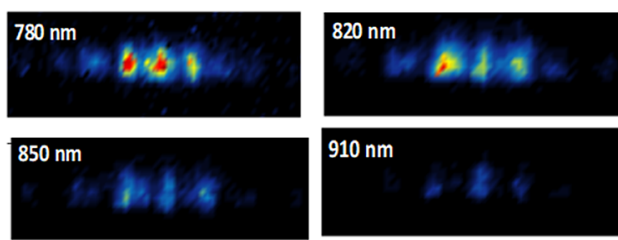

$910 \mathrm{~nm}$ d

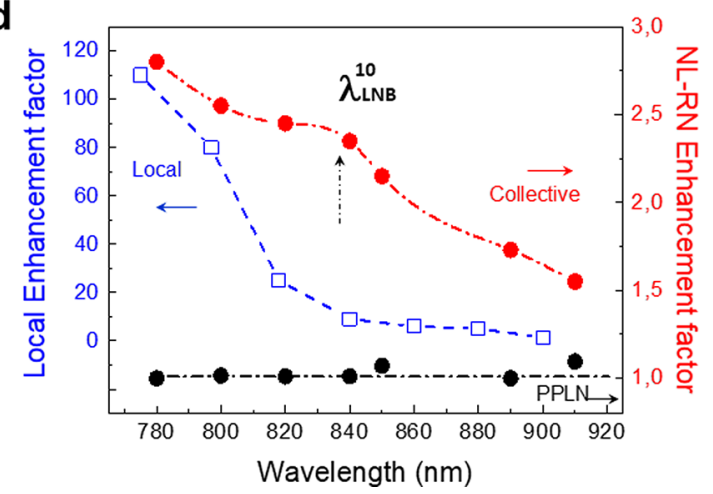

Figure 2. Optical response of $1 \mathrm{D}$ hybrid plasmonic-NLPC. (a) NL RN diffraction pattern in the absence (left) and in the presence (right) of the arrays of plasmonic chains. Top panels: fundamental beam polarized parallel to chains. Bottom panels: fundamental beam polarized perpendicular to chains. (b) Numerically simulated extinction spectrum of the plasmonic lattice deposited on the surface of the $\mathrm{LiNbO}_{3}-\mathrm{NLPC}$ (red line) and of a single plasmonic chain in air (blue). For the sake of comparison, the spectra were normalized. The absorption spectrum of $\mathrm{LiNbO}_{3}$ is also included (black line). Dashed lines correspond to the position of different RA. The spectral position of SLRs is also indicated. (c) NLRN diffraction patterns obtained from the hybrid system for different fundamental wavelengths. (d) Enhancement factor of the NL RN diffraction pattern (red dots) in the spectral region of the fundamental wavelength. The spectral dependence of the RN intensity obtained from the bare NLPC crystal is also depicted (black dots). The localized SHG enhancement measured in the vicinity of a single metallic chain is also shown (blue squares). Dashed lines are a guide for the eye.

observed at $\beta_{1}= \pm 3.8^{\circ}$ and $\beta_{2}= \pm 7.8^{\circ}$ for the first and second order, respectively, in agreement with the $\chi^{(2)}$ modulation period of our NLPC, $\Lambda=6.2 \mu \mathrm{m}$. The NL conversion efficiency was estimated to be about $10^{-6}$, in agreement with previous works. $^{48}$

To analyze the collective response in the hybrid plasmonicNLPC, we will focus on the NL RN diffraction, since the lack of transverse phase-matching in CSHG prevents the observation of any effect associated with the plasmonic lattice. Figure 2a compares the NL RN diffraction patterns obtained in the absence (left panel) and in the presence of the array of metallic chains (right panel). The results are shown for two different polarization states of the fundamental beam: parallel and perpendicular to the plasmonic chains. As observed, in the absence of plasmonic chains, the diffraction patterns obtained for fundamental waves polarized parallel and perpendicular to the chains display similar intensities, in agreement with the symmetry of the NL $\chi^{(2)}$ tensor that governs the process. ${ }^{49-51}$ However, the $\mathrm{RN}$ diffraction pattern generated in the presence of the plasmonic arrays features a marked polarization dependence. Specifically, the plasmonic lattice significantly enhances the SHG RN diffraction pattern when the fundamental beam is polarized parallel to the chains. When the fundamental beam is polarized perpendicular to the chains, the intensity of the RN pattern is similar to that obtained in the absence of plasmonic nanostructures. Here, it is interesting to note that the positions of the spots in the RN pattern are not modified by the presence of the plasmonic lattice, which indicates that the SHG enhancement takes place at the same angular position as those obtained in the bare PPLN crystal. This is consistent with the fact that the spatial period of the $\chi^{(2)}$ modulation and that of the plasmonic lattice commensurate.

The intensification of the NL RN diffracted pattern can be explained by the presence of multiple SLRs originated from the long-range coupling between the longitudinal LSP resonance supported by the metallic chains and the diffraction modes of the plasmonic lattice. ${ }^{52}$ Specifically, when the diffracted waves travel at a grazing angle on the sample surface (Rayleigh anomaly, RA), they interact with a number of plasmonic chains, and their interference with the LSP yields the emergence of SLRs located at the low-energy side of the main LSP resonance. ${ }^{21-23}$ Figure $2 b$ shows the numerically calculated extinction spectrum of our plasmonic lattice (red line). For the simulation, the plasmonic chains were periodically placed along the $x$-axis on top of a semi-infinite substrate with a refractive index of $n=2.25$ (that of $\mathrm{LiNbO}_{3}$ at the fundamental wavelengths of our experiments). ${ }^{53}$ The period of the plasmonic lattice was fixed at $\Lambda^{\prime}=3.1 \mu \mathrm{m}$, and the polarization was kept parallel to the chains. Dashed lines in the figure correspond to the theoretical position of the $m$-th order of RA calculated by: ${ }^{54}$

$$
\lambda_{\text {RA_air }}=\frac{\Lambda^{\prime}}{m}\left[1 \pm \sin \theta_{\text {in }}\right] \lambda_{\text {RA_LNB }}=\frac{\Lambda^{\prime}}{m}\left[n \pm \sin \theta_{\text {in }}\right]
$$

where $\lambda_{\mathrm{RA} \text { air }}$ and $\lambda_{\mathrm{RA} \text { LNB }}$ stand for the RA in air and substrate, respectively, $\Lambda^{\prime}$ is the plasmonic lattice periodicity, $n$ is the substrate refractive index, $m$ is an integer number related to the diffraction order, and $\theta_{\text {in }}$ is the angle of incidence. For the sake of comparison, the extinction spectrum of a single plasmonic chain calculated in a homogeneous environment (air) for light polarized parallel to the chains is also included (blue line). It 

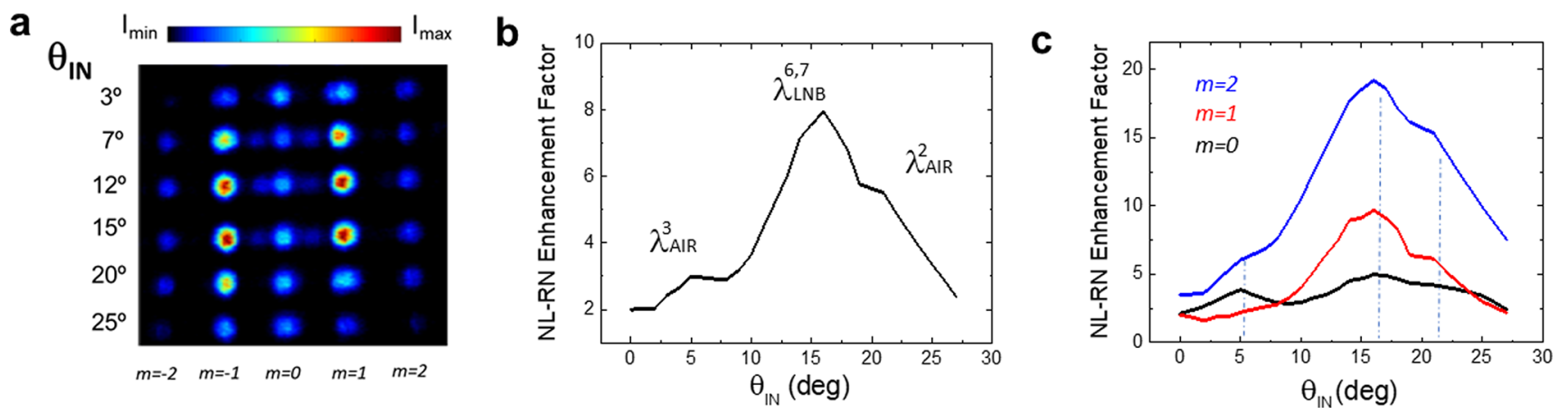

Figure 3. Angular dependence of the NL diffraction enhancement. (a) RN diffraction patterns in the presence of metallic chains for different angles of incidence. The fundamental wavelength was fixed at $1064 \mathrm{~nm}$. (b) SHG enhancement factor, estimated as the ratio between the total integrated intensity of the patterns with and without metallic chains, as a function of the angle of incidence. The different SLRs involved are indicated. (c) Enhancement factor measured for the zero (black line), first (red line), and second (blue line) orders of the NL diffraction as a function of the angle of incidence.

corresponds to the longitudinal and broad LSP centered at around $600 \mathrm{~nm}$, overlapping the fundamental and SHG frequencies used in this work.

The spectrum of the plasmonic lattice displays a broad band centered at around $600 \mathrm{~nm}$, close to that of a single plasmonic chain. Additionally, it reveals the presence of different RA orders and SLRs in the near-infrared spectral region. As marked in Figure 2b, up to five SLRs for $m=11,10,9,8$, and 7 emerge in the 700-1200 nm spectral range. They appear at $\lambda_{\mathrm{LNB}}^{11}=742 \mathrm{~nm}, \lambda_{\mathrm{LNB}}^{10}=822 \mathrm{~nm}, \lambda_{\mathrm{LNB}}^{9}=892 \mathrm{~nm}, \lambda_{\mathrm{LNB}}^{8}=1003$ and $\lambda_{\mathrm{LNB}}^{7}=1120 \mathrm{~nm}$, respectively. Three additional air resonances for $m=5,4$, and 3 are also sustained by the lattice, their spectral position overlapping the $\lambda_{\mathrm{LNB}}^{11}, \lambda_{\mathrm{LNB}}^{9}$ and $\lambda_{\mathrm{LNB}}^{8}$ substrate modes. The extinction spectrum was also calculated when the incident light is polarized perpendicular to the chain axis (Figure S3 in the Supporting Information). In this case, we obtain a negligible response of the SLRs in the spectral region of interest in agreement with the SHG results. Numerical simulations have also been used to analyze the modifications in the near field distributions in the $x-y$ and $x-z$ planes due to the presence of the SLRs in our system with respect to the case of a single plasmonic chain. The results are shown in the Supporting Information (Figure S4) and show that the field distribution is enhanced both around and in between the chains.

Figure $2 \mathrm{c}$ shows some representative NL RN diffraction patterns generated by the hybrid system when the fundamental beam is tuned in the 780-910 $\mathrm{nm}$ spectral range. The intensity of the patterns reveals a marked dependence on the fundamental wavelength. Figure $2 \mathrm{~d}$ shows the spectral dependence of the NL enhancement factor of the $\mathrm{RN}$ diffraction patterns generated when the sample was illuminated at normal incidence with a spot size of about $100 \mu \mathrm{m}$ (red dots) as a function of the fundamental beam wavelength (NL excitation spectrum). This dependence is compared to that obtained for the localized SHG enhancement observed in the vicinities of a single plasmonic chain (blue squares) by means of confocal microscopy. In both cases, the polarization of the fundamental beam was parallel to the plasmonic chains. It is important to note the flat spectral response of the NL RN pattern produced by the NLPC in the absence of metallic nanostructures (black dots).

As observed, on one hand, the localized SHG enhancement in the vicinities of a single plasmonic chain decreases when the fundamental beam is detuned from the LSP resonance of the chain (Figure 2b), in agreement with previous results pointing out the dominant role of the fundamental beam enhancement in the localized SHG intensification. ${ }^{14,15}$ On the other hand, the collective $\mathrm{RN}$ diffraction pattern clearly shows a spectral shoulder at around $840 \mathrm{~nm}$, which is consistent with the spectral position of the $\lambda_{\mathrm{LNB}}^{10}=822 \mathrm{~nm}$ SLR observed in the extinction spectrum (Figure 2b). Several factors, including slight deviations from periodicity at the illuminated area, the broad spectral line width of the femtosecond laser source used for excitation, and small deviations from the normal incidence condition in our experimental setup, can account for the $18 \mathrm{~nm}$ mismatch between theory and experiments. Larger SHG enhancement could be expected by setting the fundamental wavelength at around $740 \mathrm{~nm}$ that, unfortunately, is out of the limit of our experimental setup. In addition, given the broad spectral extension in which the different SLRs are observed $(700-1200 \mathrm{~nm})$, a spectral tunability in the optical region beyond $300 \mathrm{~nm}$ can be anticipated. As compared to other systems based on regular periodic arrangements of metallic nanostructures supporting multiple resonances, ${ }^{55,56}$ the SLRs in our system display a much broader spectral line width with the subsequent reduction in the total intensity. In addition to the slight deviations of the periodicity, the spectral broadening can be explained in terms of both, the size of the plasmonic units and the asymmetric (nonuniform refractive index) environment of our system. ${ }^{57}$ In this respect, a homogeneous index environment would probably result in a stronger diffractive coupling leading to narrower SLRs resonances and therefore more intense field enhancement. ${ }^{58,59}$ An alternative to further improve the local field enhancement includes NLPC with a submicrometer poling period matching the first order lattice mode. However, such a short periodicity would prevent the access to higher-order Bragg modes, which are of interest to expand the tunability of the NL process.

To analyze the possibility of directional tuning of the NL enhancement due to the presence of multiple SLRs in the system, we have fixed the fundamental beam at $1064 \mathrm{~nm}$ and varied the angle of incidence.

Figure 3a shows the evolution of the $\mathrm{RN}$ diffraction patterns for different angles of incidence. As aforementioned, the angular position of the $\mathrm{RN}$ spots was not altered in the presence of the plasmonic lattice. However, at specific angles of incidence, a SHG enhancement of around 1 order of magnitude was observed. Figure $3 \mathrm{~b}$ shows the enhancement factor calculated as the ratio between the integrated intensity 
with and without metallic chains as a function of the angle of incidence, $\theta$. The maximum enhancement values occur at $\theta$ angles of around $5^{\circ}, 15^{\circ}$, and $20^{\circ}$. These values are close to the angles of incidence that satisfies the RA condition on the air side for $m=2$ and 3 and on the substrate side for $m=6$ and 7, which confirms the participation of different SLRs on the enhanced NL diffraction. Thus, the collective coupling of LSP via SLRs provides a means to enhance the transverse condition of NL diffraction due to the superposition of the plasmonic lattice and that of the $\chi^{(2)}$ modulation.

Additional information on the NL optical performance of the hybrid system can be obtained by analyzing the enhancement factor of the zero, first, and second orders of the NL RN diffracted waves as a function of the angle of incidence. The results are shown in Figure 3c. As observed, the enhancement of the zero order is substantially less intense than those corresponding to the RN diffracted beams at first and second orders. This behavior can be accounted for in terms of the conversion efficiency of the SHG process. Note that unlike the zero-order, the first and second order diffracted beams satisfy the transverse QPM condition provided by the spatial modulation of $\chi^{(2)}$, being more efficiently generated when the SLRs matches the fundamental radiation wavelength. Additionally, we observe that the enhancement of the second order NL RN diffracted beam is approximately twice that of the first order. This effect can be due to the additional diffractive coupling of the localized enhanced SHG produced by each of the single plasmonic chain at each domain boundary (see Figure 1a). In fact, the plasmonic lattice periodicity $\left(\Lambda^{\prime}=3.1\right.$ $\mu \mathrm{m})$ allows the diffraction of the SHG photons enhanced at the domain walls with deflection angles that coincide with the even orders of the $\mathrm{RN}$ diffraction pattern generated by the spatial modulation of $\chi^{(2)}(\Lambda=6.2 \mu \mathrm{m})$.

Finally, to emphasize the potential of combining engineered $\chi^{(2)}$-based NLPC with periodical arrays of plasmonic nanostructures, we extended the study to a $2 \mathrm{D}$ configuration. The 2D NLPC studied in this work consists of a square lattice of hexagonal inverted ferroelectric domains in $\mathrm{LiNbO}_{3}$, with domain sizes and separation distances of around $1.5 \mu \mathrm{m}$. The extension of the QPM conditions from $1 \mathrm{D}$ to $2 \mathrm{D} \chi^{(2)}$ modulation allows access to several reciprocal lattice vectors, leading to a different spatial distribution of the SHG waves. $^{2,4,12}$ Accordingly, as shown in Figure 4a, conical CSHG and NL RN at pairs of diffraction orders $(m, n)$ reflecting the $2 \mathrm{D}$ symmetry of the reciprocal lattice can be distinguished.

The combination of Ag NPs with the 2D-NLPC results into the formation of micrometric hexagonal plasmonic necklaces arranged in a square lattice with the same periodicity as that displayed by the NL structure $\Lambda_{2 \mathrm{D}} \sim 3 \mu \mathrm{m}$ (Figure $4 \mathrm{~b}$ ). Moreover, the separation between the hexagon sides, $\Lambda_{2 \mathrm{D}}^{\prime} \sim$ $1.5 \mu \mathrm{m}$, also commensurate with the $\chi^{(2)}$ spatial modulation. As for the $1 \mathrm{D}$ case, the hexagonal necklaces support a broad and intense LSP extending from the VIS to the NIR region, which spectrally overlaps the optical fields involved in our experiments. ${ }^{60}$ As demonstrated, they also exhibit an extraordinary localized SHG enhancement (up to 400 times) due to the coupling of the plasmonic modes of the necklaces with the fundamental radiation. ${ }^{15}$ The expanded view of the central NL pattern displayed in Figure $4 \mathrm{c}$ highlights the multidirectional enhancement of the RN SHG radiation in the presence of periodical arrays of hexagonal necklaces (right panel). In Figure $4 \mathrm{~d}$ we show the spectral dependence of the enhanced
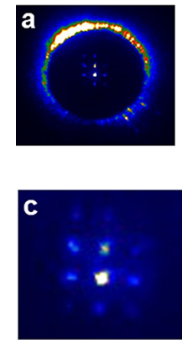

$\mathrm{LiNbO}_{3}$
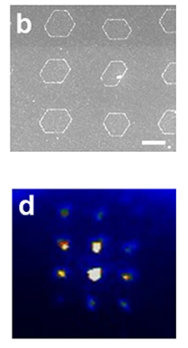

$\mathrm{Ag} / \mathrm{LiNbO}_{3}$

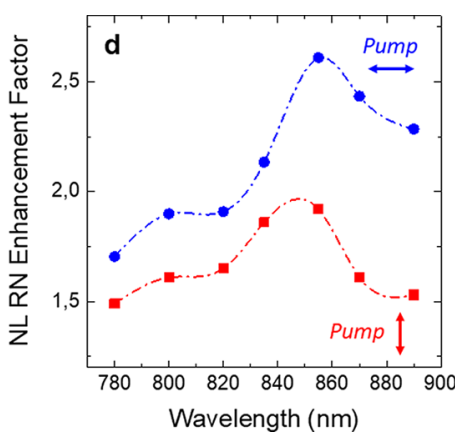

Figure 4. Nonlinear diffraction in a 2D hybrid plasmonic-NLPC. (a) Far-field SHG pattern showing the conical SHG distribution obtained for the CSHG. The central region shows the spots corresponding to the multiorder NL RN diffraction. (b) SEM image of a square lattice of hexagonal plasmonic necklaces of Ag NPs formed on the domain boundary surfaces of inverted hexagonal ferroelectric domains in $\mathrm{LiNbO}_{3}$. The scale bar is $1 \mu \mathrm{m}$. (c) Detail of the NL RN diffraction patterns obtained without (left) and with (right) plasmonic nanostructures for a fundamental wavelength of $800 \mathrm{~nm}$. (d) Integrated RN SHG enhancement factor as a function of the fundamental wavelength at normal incidence for two different linear polarization states of the fundamental beam, parallel to the $x$-axis (blue dots) and perpendicular to it ( $y$-axis, red dots). Dashed lines are a guide for the eye.

NL process recorded at normal incidence for two different polarization states of the fundamental beam, parallel to the $x$ axis (blue dots) and perpendicular to it ( $y$-axis, red dots). As observed, the slight variations of the lattice periodicity along the $x$ and $y$ directions, spectrally shift the corresponding RA at the fundamental wavelength. In particular, the coupling of the LSP modes sustained by the necklaces to the $m=10$ Bragg mode in the square plasmonic lattice $(\langle 10,0\rangle$ and $\langle 0,10\rangle$ for $x$ and $y$ polarizations, respectively) leads to the emergence of a SLR centered at around $850 \mathrm{~nm}$. We also note the presence of a much weaker resonance centered at around $800 \mathrm{~nm}$, which could be attributable to the fifth order diffraction mode of the square plasmonic lattice on air. The similarity of the results obtained for orthogonal pumping polarizations is consistent with both the far-field plasmonic response supported by the necklaces, which is polarization independent, ${ }^{60}$ and the square arrangement of the plasmonic lattice. The measured enhancement factor is comparable to that obtained for the $1 \mathrm{D}$ lattice for the same experimental conditions (Figure 2d) and could be improved by finely tuning the lowest order SLRs under oblique incidence. It is worth mentioning that the spectral features are better resolved in our $2 \mathrm{D}$ system than in the $1 \mathrm{D}$ one, which is related to the larger number of illuminated patterns.

\section{SUMMARY AND CONCLUSIONS}

To summarize, we have experimentally demonstrated the ability of plasmonic lattices composed by periodical arrays of metallic chains to enhance quasi-phase matched NL diffraction processes in a NL crystal with a spatial modulation of $\chi^{(2)}$. The plasmonic lattice supports multiple SLRs at the technologically relevant NIR spectral region providing a new path for generating and control coherent light with broad spectral tunability in miniaturized optical devices. When combined with NLPCs, the excitation of SLRs at the fundamental frequency allows to enhance the directional SHG originated in the NL system in around 1 order of magnitude. The enhancement is achieved without the need of refractive index 
matching and displays a broad spectral tunability which can be of interest for in vivo sensing applications where an air (or water) superstrate can be needed.

In addition, due to the plasmonic field localization around the nanostructures, the superlattice also exploits the intriguing nonlinearity of ferroelectric domain walls, enabling additional routes for the generation of SHG processes at the nanoscale that collectively contribute to boost the coherent radiated waves at well-defined directions. The results further expand the potential of plasmonic lattices for light manipulation and control and can be extended to other spatial configurations provided by NLPCs such as vortex beam generation for the nonlinear manipulation of orbital angular momentum. In fact, the integration of the functional properties of NLPC with engineered arrays of plasmonic nanostructures opens interesting prospects for the development of new integrated platforms with an active control on the directional NL response. Finally, it is worth mentioning the potential for increasing the plasmonic enhancement of the $\chi^{(2)}$-based NL process by finely engineering the period of the NLPC at submicrometer scales and by optimizing the $Q$ factor of the SLRs in a homogeneous index matched configuration.

\section{MATERIALS AND METHODS}

Sample Preparation. The ferroelectric patterns were fabricated by using direct electron beam writing. The inverted domains cross the whole sample thickness, the size and shape of the irradiated areas being the same at both crystal faces. Details on the fabrication procedure can be found elsewhere. $^{61,62}$ The crystal thickness was about $500 \mu \mathrm{m}$ and the surface was polished up to optical grade. The hybrid plasmonic-NLPCs were fabricated by means of ferroelectric lithography. ${ }^{40}$ Periodically poled $\mathrm{LiNbO}_{3}$ structures were immersed into an $\mathrm{AgNO}_{3}$ solution $\left(0.01 \mathrm{M}\right.$ ) at $60{ }^{\circ} \mathrm{C}$, while their polar surface was irradiated with above bandgap UV light ( $\mathrm{Hg}$ pen-lamp at $253.6 \mathrm{~nm}$ ) for $4 \mathrm{~min}$. The average NP size and interparticle distance were estimated from high-resolution images obtained by a scanning electron microscope (Philips XL30 SFEG). The dark-field images were obtained in transmission mode by means of an Olympus BX51 microscope equipped with a dark-field condenser.

Optical Characterization. Confocal SHG Experiments. The SHG scans were recorded on a customized confocal microscope (Olympus BX41) provided with a XY motorized stage driven by Labspec software. A tunable femtosecond Ti:sapphire laser (3900S Tsunami Spectra Physics) delivering $100 \mathrm{fs}$ pulses at a repetition rate of $80 \mathrm{MHz}$ was used as a fundamental beam, and focused onto the $\mathrm{LiNbO}_{3}$ surface by a $100 \times$ microscope objective (Olympus, N.A. 0.9) The SHG signal was collected in backscattering geometry with the same objective and fiber-coupled into a Peltier cooled photomultiplier tube (Hamamatsu R636). The image contrast was obtained by plotting the SHG integrated intensity recorded at each step (pixel). To avoid damaging the structures, the incident pump power was kept below $1 \mathrm{~mW}$. Half-wave plates were used to control the laser polarization.

Nonlinear Diffraction. The nonlinear diffraction experiments were performed with the fundamental beam traveling parallel to the ferroelectric axis. The laser beam was linearly polarized and launched into the surface with a spot diameter of about $100 \mu \mathrm{m}$. The input power was $5 \mathrm{~mW}$. The multiorder nonlinear diffraction patterns generated by the nonlinear system were projected on a screen and imaged with an
ANDOR Zyla sCMOS camera. The spectral dependence of the NL RN diffraction process obtained at normal incidence using a tunable femtosecond Ti:shappire laser (Spectra Physics Model 177-Series) delivering $100 \mathrm{fs}$ pulses at a repetition rate of $80 \mathrm{MHz}$ as a fundamental beam. For the angular analysis, the sample was mounted on a rotating stage. As a fundamental radiation, a picosecond $\mathrm{Nd}$ :YAG laser operating at $1064 \mathrm{~nm}$ with a nominal repetition rate of $35 \mathrm{kHz}$ was used.

Numerical Calculations. Three-dimensional finite-difference time-domain (FDTD) simulations were performed employing the software FDTD Solutions from Lumerical Solutions. Arrays of silver NP chains on top of a semi-infinite $\mathrm{LiNbO}_{3}$ substrate were illuminated with a plane wave polarized parallel to the chains axis. Silver dielectric function fitting the optical data from Palik was used for the plasmonic NPs, ${ }^{63}$ and a refractive index $n=2.25$ was set to characterize optically the $\mathrm{LiNbO}_{3}$ substrate. ${ }^{53}$ Spherical NPs of diameter $d=55 \mathrm{~nm}$, with a $2 \mathrm{~nm}$ gap between consecutive particles, were considered. Periodic boundary conditions were applied along the $x$ and $y$ directions of the 3D simulation box, and perfectly matching layer (PML) conditions were imposed on the boundary along the plane wave injection, taking one unit cell in the simulations. Specifically, to get converged results, the size of the simulation box along the $z$-axis was fixed to $2 \mu \mathrm{m}$, and a uniform mesh type with a maximum mesh step of $1 \mathrm{~nm}$ was needed, setting the mesh refinement to "conformal variant 1 ". Finally, reflectance $(R)$ and transmittance $(T)$ results were obtained, accordingly, from frequency-domain field monitors at the illumination (air) and transmission $\left(\mathrm{LiNbO}_{3}\right.$ substrate) regions.

\section{ASSOCIATED CONTENT}

Supporting Information

The Supporting Information is available free of charge at https://pubs.acs.org/doi/10.1021/acsphotonics.1c00778.

Sample specifications; SLRs in 1D plasmonic superlattice; Angular dependence of multiorder nonlinear Raman Nath diffraction at oblique incidence (PDF)

\section{AUTHOR INFORMATION}

\section{Corresponding Author}

Mariola O Ramírez - Departamento Física de Materiales, Instituto de Materiales Nicolás Cabrera and Condensed Matter Physics Center (IFIMAC), Universidad Autónoma de Madrid, 28049 Madrid, Spain; (1) orcid.org/0000-00021233-1769; Email: mariola.ramirez@uam.es

\section{Authors}

Alejandro Gómez-Tornero - Departamento Física de Materiales, Instituto de Materiales Nicolás Cabrera and Condensed Matter Physics Center (IFIMAC), Universidad Autónoma de Madrid, 28049 Madrid, Spain

Pablo Palacios - Departamento Física de Materiales, Instituto de Materiales Nicolás Cabrera and Condensed Matter Physics Center (IFIMAC), Universidad Autónoma de Madrid, 28049 Madrid, Spain

Pablo Molina - Departamento Física de Materiales, Instituto de Materiales Nicolás Cabrera and Condensed Matter Physics Center (IFIMAC), Universidad Autónoma de Madrid, 28049 Madrid, Spain

Sol Carretero-Palacios - Departamento Física de Materiales, Instituto de Materiales Nicolás Cabrera and Condensed 
Matter Physics Center (IFIMAC), Universidad Autónoma de Madrid, 28049 Madrid, Spain; (1) orcid.org/0000-00019915-5050

Luisa E. Bausá - Departamento Física de Materiales, Instituto de Materiales Nicolás Cabrera and Condensed Matter Physics Center (IFIMAC), Universidad Autónoma de Madrid, 28049 Madrid, Spain; (1) orcid.org/0000-00028744-213X

Complete contact information is available at:

https://pubs.acs.org/10.1021/acsphotonics.1c00778

\section{Notes}

The authors declare no competing financial interest.

\section{ACKNOWLEDGMENTS}

This work has been supported by the Spanish Government (Contracts MAT2016-76106-R and PID2019-108257GB-I00/ AEI/10.13039/501100011033 and María de Maeztu "Programme for Units of Excellence in R\&D CEX2018-000805-M) and Comunidad de Madrid (Grant SI1/PJI/2019-00105).

\section{REFERENCES}

(1) Freund, I. Nonlinear Diffraction. Phys. Rev. Lett. 1968, 21, 1404. (2) Berger, V. Nonlinear Photonic Crystals. Phys. Rev. Lett. 1998, 81, 4136.

(3) Hu, X.; Zhang, Y.; Zhu, S. Nonlinear Beam Shaping in Domain Engineered Ferroelectric Crystals. Adv. Mater. 2019, 32, 1903775.

(4) Arie, A.; Voloch, N. Periodic, Quasi-Periodic, and Random Quadratic Nonlinear Photonic Crystals. Laser Photonics Rev. 2010, 4, 355-373.

(5) Keren-Zur, S.; Ellenbogen, T. A New Dimension for Nonlinear Photonic Crystals. Nat. Photonics 2018, 12, 575-577.

(6) Xu, T.; Switkowski, K.; Chen, X.; Liu, S.; Koynov, K.; Yu, H.; Zhang, H.; Wang, J.; Sheng, Y.; Krolikowski, W. Three-Dimensional Nonlinear Photonic Crystal in Ferroelectric Barium Calcium Titanate. Nat. Photonics 2018, 12, 591-595.

(7) Wei, D.; Wang, C.; Wang, H.; Hu, X.; Wei, D.; Fang, X.; Zhang, Y.; Wu, D.; Hu, Y.; Li, J.; Zhu, S.; Xiao, M. Experimental Demonstration of a Three-Dimensional Lithium Niobate Nonlinear Photonic Crystal. Nat. Photonics 2018, 12, 596-600.

(8) Wei, D.; Wang, C.; Xu, X.; Wang, H.; Hu, Y.; Chen, P.; Li, J.; Zhu, Y.; Xin, C.; Hu, X.; Zhang, Y.; Wu, D.; Chu, J.; Zhu, S.; Xiao, M. Efficient Nonlinear Beam Shaping in Three-Dimensional Lithium Niobate Nonlinear Photonic Crystals. Nat. Commun. 2019, 10, 4193.

(9) Liu, S.; Switkowski, K.; Xu, C.; Tian, J.; Wang, B.; Lu, P.; Krolikowski, W.; Sheng, Y. Nonlinear Wavefront Shaping with Optically Induced Three-Dimensional Nonlinear Photonic Crystals. Nat. Commun. 2019, 10, 3208.

(10) Ellenbogen, T.; Voloch-Bloch, N.; Ganany-Padowicz, A.; Arie, A. Nonlinear Generation and Manipulation of Airy Beams. Nat. Photonics 2009, 3, 395-398.

(11) Canalias, C.; Pasiskevicius, V. Mirrorless Optical Parametric Oscillator. Nat. Photonics 2007, 1, 459-462.

(12) Mateos, L.; Molina, P.; Galisteo, J.; López, C.; Bausá, L. E.; Ramírez, M. O. Simultaneous Generation of Second to Fifth Harmonic Conical Beams in a Two Dimensional Nonlinear Photonic Crystal. Opt. Express 2012, 20, 29940-29948.

(13) Leng, H. Y.; Yu, X. Q.; Gong, Y. X.; Xu, P.; Xie, Z. D.; Jin, H.; Zhang, C.; Zhu, S. N. On-Chip Steering of Entangled Photons in Nonlinear Photonic Crystals. Nat. Commun. 2011, 2, 429.

(14) Ramírez, M. O.; Molina, P.; Gómez-Tornero, A.; HernándezPinilla, D.; Sánchez-García, L.; Carretero-Palacios, S.; Bausá, L. E. Hybrid Plasmonic-Ferroelectric Architectures for Lasing and SHG Processes at the Nanoscale. Adv. Mater. 2019, 31, 1901428.

(15) Gomez-Tornero, A.; Tserkezis, C.; Mateos, L.; Bausá, L. E.; Ramírez, M. O. 2D Arrays of Hexagonal Plasmonic Necklaces for
Enhanced Second Harmonic Generation. Adv. Mater. 2017, 29, 1605267.

(16) Sánchez-Garcia, L.; Tserkezis, C.; Ramírez, M. O.; Molina, P.; Carvajal, J. J.; Aguilo, M.; Diaz, F.; Aizpurua, J.; Bausá, L. E. Plasmonic Enhancement of Second Harmonic Generation from Nonlinear $\mathrm{RbTiOPO}_{4}$ Crystals by Aggregates of Silver Nanostructures. Opt. Express 2016, 24, 8491-8500.

(17) Lehr, D.; Reinhold, J.; Thiele, I.; Hartung, H.; Dietrich, K.; Menzel, C.; Pertsch, T.; Kley, E.-B.; Tünnermann, A. Enhancing Second Harmonic Generation in Gold Nanoring Resonators Filled with Lithium Niobate. Nano Lett. 2015, 15, 1025-1030.

(18) Chauvet, N.; Ethis de Corny, M.; Jeannin, M.; Laurent, G.; Huant, S.; Gacoin, T.; Dantelle, G.; Nogues, G.; Bachelier, G. Hybrid KTP-Plasmonic Nanostructures for Enhanced Nonlinear Optics at the Nanoscale. ACS Photonics 2020, 7, 665-672.

(19) Li, Z.; Corbett, B.; Gocalinska, A.; Pelucchi, E.; Chen, W.; Ryan, K. M.; Khan, P.; Silien, C.; Xu, H.; Liu, N. Direct Visualization of Phase-Matched Efficient Second Harmonic and Broadband Sum Frequency Generation in Hybrid Plasmonic Nanostructures. Light: Sci. Appl. 2020, 9, 180.

(20) Timpu, F.; Hendricks, N. R.; Petrov, M.; Ni, S.; Renaut, C.; Wolf, H.; Isa, L.; Kivshar, Y.; Grange, R. Enhanced Second-Harmonic Generation from Sequential Capillarity-Assisted Particle Assembly of Hybrid Nanodimers. Nano Lett. 2017, 17 (9), 5381-5388.

(21) Wang, D.; Guan, J.; Hu, J.; Bourgeois, M. R.; Odom, T. W. Manipulating Light-Matter Interactions in Plasmonic Nanoparticle Lattices. Acc. Chem. Res. 2019, 52, 2997-3007.

(22) Wang, W.; Ramezani, M.; Vakevainen, A. I.; Torma, P.; Rivas, J. G.; Odom, T. W. The Rich Photonic World of Plasmonic Nanoparticle Arrays. Mater. Today 2018, 21, 303-314.

(23) Kravets, V. G.; Kabashin, A. V.; Barnes, W. L.; Grigorenko, A. N. Plasmonic Surface Lattice Resonances: A Review of Properties and Applications. Chem. Rev. 2018, 118, 5912-5951.

(24) Wang, D.; Yang, A.; Wang, W.; Hua, Y.; Schaller, R. D.; Schatz, G. C.; Odom, T. W. Band-Edge Engineering for Controlled MultiModal Nanolasing in Plasmonic Superlattices. Nat. Nanotechnol. 2017, 12, 889-894.

(25) Lozano, G.; Rodriguez, S. RK.; Verschuuren, M. A.; Gómez Rivas, J. Metallic Nanostructures for Efficient LED Lighting. Light: Sci. Appl. 2016, 5, e16080.

(26) Hooper, D. C.; Kuppe, C.; Wang, D.; Wang, W.; Guan, J.; Odom, T. W.; Valev, V. K. Second Harmonic Spectroscopy of Surface Lattice Resonances. Nano Lett. 2019, 19, 165-172.

(27) Liu, B.; Chen, S.; Zhang, J.; Yao, X.; Zhong, J.; Lin, H.; Huang, T.; Yang, Z.; Zhu, J.; Liu, S.; Lienau, C.; Wang, L.; Ren, B. A Plasmonic Sensor Array with Ultrahigh Figures of Merit and Resonance Linewidths down to $3 \mathrm{Nm}$. Adv. Mater. 2018, 30, 1706031.

(28) Keren-Zur, S.; Michaeli, L.; Suchowski, H.; Ellenbogen, A. Shaping Light with Nonlinear Metasurfaces. Adv. Opt. Photonics 2018, 10, 309-353.

(29) Czaplicki, R.; Kiviniemi, A.; Huttunen, M. J.; Zang, X.; Stolt, T.; Vartiainen, I.; Butet, J.; Kuittinen, M.; Martin, O. J. F.; Kauranen, M. Less is More: Enhancement of Second-Harmonic Generation from Metasurfaces by Reduced Nanoparticle Density. Nano Lett. 2018, 18, $7709-7714$.

(30) Hooper, D. C.; Kuppe, C.; Wang, D.; Wang, W.; Guan, J.; Odom, T. W.; Valev, V. K. Second Harmonic Spectroscopy of Surface Lattice Resonances. Nano Lett. 2019, 19, 165-172.

(31) Krasnok, A.; Tymchenko, M.; Alù, A. Nonlinear Metasurfaces: A Paradigm Shift in Nonlinear Optics. Mater. Today 2018, 21, 8-21.

(32) Wu, X.-X.; Jiang, W.-Y.; Wang, X.-F.; Zhao, L.-Y.; Shi, J.; Zhang, S.; Sui, X.; Chen, Z.-X.; Du, W.-N.; Shi, J.-W.; Liu, Q.; Zhang, Q.; Zhang, Y.; Liu, X.-F. Inch-Scale Ball-In-Bowl Plasmonic Nanostructure Arrays for Polarization-Independent Second-Harmonic Generation. ACS Nano 2021, 15, 1291-1300.

(33) Kong, Y.; Bo, F.; Wang, W.; Zheng, D.; Liu, H.; Zhang, G.; Rupp, R.; Xu, J. Recent Progress in Lithium Niobate: Optical Damage, Defect Simulation, and On-Chip Devices. Adv. Mater. 2020, $32,1806452$. 
(34) Sun, D.; Zhang, Y.; Wang, D.; Song, W.; Liu, X.; Pang, J.; Geng, D.; Sang, Y.; Liu, H. Microstructure and Domain Engineering of Lithium Niobate Crystal Films for Integrated Photonic Applications. Light: Sci. Appl. 2020, 9, 197.

(35) Lin, J.; Bo, F.; Cheng, Y.; Xu, J. Advances in On-Chip Photonic Devices Based on Lithium Niobate on Insulator. Photonics Res. 2020, 8, 1910-1936.

(36) Boes, A.; Corcoran, B.; Chang, L.; Bowers, J.; Mitchell, A. Status And Potential Of Lithium Niobate on Insulator (LNOI) for Photonic Integrated Circuits. Laser Photonics Rev. 2018, 12, 1700256.

(37) Hickstein, D. D.; Carlson, D. R.; Kowligy, A.; Kirchner, M.; Domingue, S. R.; Nader, N.; Timmers, H.; Lind, A.; Ycas, G. G.; Murnane, M. M.; Kapteyn, H. C.; Papp, S. B.; Diddams, S. A. HighHarmonic Generation in Periodically Poled Waveguides. Optica. 2017, 4, 1538-1544.

(38) Myers, L. E.; Eckardt, R. C.; Fejer, M. M.; Byer, R. L.; Bosenberg, W. R. Multigrating Quasi-Phase-Matched Optical Parametric Oscillator in Periodically Poled $\mathrm{LiNbO}_{3}$. Opt. Lett. 1996, 21, 591-593.

(39) An, N.; Ren, H.; Zheng, Y.; Deng, X.; Chen, X. Conical Second Harmonic Generation In One-Dimension Nonlinear Photonic Crystal. Appl. Phys. Lett. 2012, 100, 221103.

(40) Kalinin, S. V.; Bonnell, D. A.; Alvarez, T.; Lei, X. J.; Hu, Z. H.; Shao, R.; Ferris, J. H. Ferroelectric Lithography of Multicomponent Nanostructures. Adv. Mater. 2004, 16, 795-799.

(41) Yraola, E.; Sánchez-García, L.; Tserkezis, C.; Molina, P.; Ramírez, M. O.; Plaza, J. L.; Aizpurua, J.; Bausá, L. E. Controlling Solid State Gain Media by Deposition of Silver Nanoparticles: From Thermally-Quenched to Plasmon-Enhanced $\mathrm{Nd}^{3+}$ Luminescence. Opt. Express 2015, 23, 15670-15679.

(42) Yraola, E.; Sánchez-García, L.; Tserkezis, C.; Molina, P.; Ramírez, M. O.; Aizpurua, J.; Bausá, L. E. Polarization-Selective Enhancement of $\mathrm{Nd}^{3+}$ Photoluminescence Assisted by Linear Chains of Silver Nanoparticles. J. Lumin. 2016, 169, 569-573.

(43) Hernández-Pinilla, D.; Molina, P.; de las Heras, C.; BravoAbad, J.; Bausá, L. E.; Ramírez, M. O. Multiline Operation from a Single Plasmon-Assisted Laser. ACS Photonics 2018, 5, 406-412.

(44) Molina, P.; Yraola, E.; Ramírez, M. O.; Tserkezis, C.; Plaza, J. L.; Aizpurua, J.; Bravo-Abad, J.; Bausá, L. E. Plasmon-Assisted $\mathrm{Nd}^{3+}$ Based Solid-State Nanolaser. Nano Lett. 2016, 16, 895-899.

(45) Saltiel, S. M.; Sheng, Y.; Voloch-Bloch, N.; Neshev, D. N.; Krolikowski, W.; Arie, A.; Koynov, K.; Kivshar, Y. S. Cerenkov-Type Second-Harmonic Generation In Two-Dimensional Nonlinear Photonic Structures. IEEE J. Quantum Electron. 2009, 45, 1465-1472.

(46) Saltiel, S. M.; Neshev, D. N.; Krolikowski, W.; Arie, A.; Bang, O.; Kivshar, Y. S. Multiorder Nonlinear Diffraction in Frequency Doubling Processes. Opt. Lett. 2009, 34, 848-850.

(47) Sheng, Y.; Kong, Q.; Wang, W.; Kalinowski, K.; Krolikowski, W. Theoretical Investigations of Nonlinear Raman-Nath Diffraction in The Frequency Doubling Process. J. Phys. B: At., Mol. Opt. Phys. 2012, 45, 055401.

(48) Vyunishev, A. M.; Slabko, V. V.; Baturin, S.; Akhmatkhanov, A. R.; Shur, V. Ya. Nonlinear Raman-Nath Diffraction of Femtosecond Laser Pulses. Opt. Lett. 2014, 39, 4231-4234.

(49) Saltiel, S. M.; Neshev, D. N.; Fischer, R.; Krolikowski, W.; Arie, A.; Kivshar, Y. S. Generation Of Second-Harmonic Conical Waves Via Nonlinear Bragg Diffraction. Phys. Rev. Lett. 2008, 100, 103902.

(50) Sheng, Y.; Saltiel, S. M.; Krolikowski, W.; Arie, A.; Koynov, K.; Kivshar, Y. S. Cerenkov-Type Second-Harmonic Generation with Fundamental Beams of Different Polarizations. Opt. Lett. 2010, 35, 1317-1319.

(51) Molina, P.; Ramírez, M. O.; Garcia, B. J.; Bausá, L. E. Directional Dependence of the Second Harmonic Response in TwoDimensional Nonlinear Photonic Crystals. Appl. Phys. Lett. 2010, 96, 261111.

(52) Liu, S.; Yue, P.; Zhang, S.; Wang, M.; Dai, H.; Chen, Y.; Nie, Z.; Cui, Y.; Han, J.; Duan, H. Metasurfaces Composed Of Plasmonic Molecules: Hybridization Between Parallel and Orthogonal Surface Lattice Resonances. Adv. Opt. Mater. 2020, 8, 1901109.
(53) Zelmon, D. E.; Small, D. L.; Jundt, D. Infrared Corrected Sellmeier Coefficients for Congruently Grown Lithium Niobate and 5 Mol. \% Magnesium Oxide-Doped Lithium Niobate. J. Opt. Soc. Am. B 1997, 14, 3319-3322.

(54) Kravets, V. G.; Schedin, F.; Grigorenko, A. N. Extremely Narrow Plasmon Resonances Based on Diffraction Coupling of Localized Plasmons in Arrays of Metallic Nanoparticles. Phys. Rev. Lett. 2008, 101, 087403.

(55) Reshef, O.; Saad-Bin-Alam, M.; Huttunen, M. J.; Carlow, G.; Sullivan, B. T.; Ménard, J. M.; Dolgaleva, K.; Boyd, R. W. Multiresonant High-Q Plasmonic Metasurfaces. Nano Lett. 2019, 19, 6429-6434.

(56) Wang, D.; Yang, A.; Hryn, A. J.; Schatz, G. C.; Odom, T. W. Superlattice Plasmons in Hierarchical Au Nanoparticle Arrays. ACS Photonics 2015, 2, 1789-1794.

(57) Cherqui, C.; Bourgeois, M. R.; Wang, D.; Schatz, G. C. Plasmonic Surface Lattice Resonances: Theory and Computation. Acc. Chem. Res. 2019, 52, 2548-2558.

(58) Auguie, B.; Bendaña, X.; Barnes, W. L.; García de Abajo, F. Diffractive Arrays of Gold Nanoparticles near an Interface: Critical Role of the Substrate. Phys. Rev. B: Condens. Matter Mater. Phys. 2010, $82,155447$.

(59) Bin-Alam, M. S.; Reshef, O.; Mamchur, Y.; Alam, M. Z.; Carlow, G.; Upham, J.; Sullivan, B. T.; Ménard, J. M.; Huttunen, M. J.; Boyd, R. W.; Dolgaleva, K. Ultra-High-Q Resonances in Plasmonic Metasurfaces. Nat. Commun. 2021, 12, 974.

(60) Gómez-Tornero, A.; Tserkezis, C.; Moreno, J. R.; Bausá, L. E.; Ramírez, M. O. Field Enhancement and Spectral Features of Hexagonal Necklaces of Silver Nanoparticles for Enhanced Nonlinear Optical Processes. Opt. Express 2018, 26, 22394-22404.

(61) Mateos, L.; Bausá, L. E.; Ramírez, M. O. Two Dimensional Ferroelectric Domain Patterns in $\mathrm{Yb}^{3+}$ Optically Active $\mathrm{LiNbO}_{3}$ Fabricated By Direct Electron Beam Writing. Appl. Phys. Lett. 2013, 102, 042910.

(62) Mateos, L.; Bausá, L. E.; Ramírez, M. O. Micro-spectroscopic characterization of ferroelectric domain structures in $\mathrm{Yb}^{3+}: \mathrm{LiNbO}_{3}$ prepared by electron beam writing. Opt. Mater. Express 2014, 4, 1077-1087.

(63) Palik, E. D. Handbook of Optical Constants of Solids; Academic Press, 1985. 